\title{
Performance of Heuristic Priority Rule for Multiskilled Resource-Constrained Scheduling
}

\author{
Narongrit Wongwai ${ }^{1}$, Suphawut Malaikrisanachalee ${ }^{2}$, Jukkrit Prajongmoon ${ }^{3}$ \\ ${ }^{1}$ Faculty of Engineering at Sriracha, Kasetsart University Sriracha Campus, Chonburi 20230, Thailand, \\ narongrit@eng.src.ku.ac.th \\ ${ }^{2}$ Faculty of Engineering, Kasetsart University, Bangkok 10300, Thailand, fengshm@ku.ac.th \\ ${ }^{3}$ Thailand Regional Irrigation Office 10, Royal Irrigation Department, Sing Buri 16000, Thailand, \\ juk.pra@gmail.com
}

\begin{abstract}
Nowadays, without a classification system that helps the project manager to decide which heuristic applies when mitigating the multiskilled resource-constrained scheduling, they must try several of rules until they find one that compares favorably (shortest duration) with the results of the other heuristic priority rules. This study explored the twenty-three existing heuristics' performance for multiskilled resource-constrained scheduling. The results found that the heuristics with good performance are TIMROS, TIMRES, ACROS, WCS ACS and ACTRES. Overall, they outperform the others in shortening the project duration. It should be pointed out that the heuristics dealing with the use of several information are likely better to get shorter project duration. There are enough statistical evidences to conclude that their criterions have a significant effect on reducing project duration by approximately 1-2 times of the standard deviation. The top four heuristics: TIMROS, TIMRES, ACROS and ACTRESS classified into the composite rule produced the lowest average of project duration. It is also found that Serial Schedule Scheme (SSS) underperform the Partial Schedule Scheme (PSS). This study has the contribution for the project managers to decide which heuristic applies when mitigating the multiskilled resource over allocation problem in term of minimum project duration.
\end{abstract}

Key words: heuristic priority rules, multiskilled resource-constrained scheduling, project duration, resource over allocation

\section{INTRODUCTION}

The heuristic method can be defined as the method that facilitates the process of finding the solution by means of a rule [1]. The heuristic method is one of the scheduling methods used for the resource constrained projects. The limited resource project scheduling problem falls under combinatorial problems. The limitations of optimization approach are not successful on these combinatorial problems: several heuristic scheduling models are developed to produce near optimal or good solutions. Heuristic priority rules have been one of the most important solution technologies for multi-resource constrained project scheduling problems. The heuristic method is used to assign rule priorities the activities that competing for the renewable resources. Although, the solution obtained from the heuristics is not guarantee optimal solution but rather produce a good feasible solution. The heuristic procedures for resource-constrained project scheduling is used a priority to rank the activities and then activities with resource limitation. In heuristic approach, a forward pass is regularly checked whether the resource demand is exactly the limits of available of resources. Some activities involved in resource conflicts and may not be delay for some time. These delay activities will enter the queue waiting for the requirement of resources available. The process repeated until all the activities are schedule in the project. The selection of which activities to be postponed is depends upon the priorities assigned to each activity. These priorities are assigned as per the heuristic rules. The objective of the priority rule is to minimize the project duration. Nowadays, without a classification system that helps project managers to decide which heuristic applies when mitigating the resource over allocation problem, they must try several of rules until they find one that compares favorably (shortest duration) with the results of the other priority rules. So far, past studies of resource-constrained scheduling heuristics by many scholars have focused on testing different sets of priority rules with paying attention to the conditions under which each heuristic produces better results i.e. shorter durations. However, none of past studies focus on testing the effectiveness of these priority rules when applying with the project which is planned under the assumption that each resource can have more than one skill and resource substitution is allowed. This study aims to reveal the heuristics' performance which has the contribution for the project managers to decide which heuristic applies when mitigating the resource over allocation problem in term of minimum project duration.

\section{HEURISTIC-BASED RESOURCE SCHEDULING}

Heuristic method is an alternative way of study to find out the solution with simple rule. The method contributes to nearly optimal solution and sometimes gives optimal solution. Because of the ability of finding out the solution 
fast, it is suitable for the complicated problems. Heuristic method is generally used for resources-constrained project scheduling. It involves in heuristic rule focusing on priority order of the activities that demand the same resources on the same day. According to the method, the resources are first given to the higher priority activities, then the resources will be given to the lower priority activities accordingly. A number of heuristic rule are the main focus of studies since 1960 and they can be classified into five categories: network-based rule, critical path-based rule, composite rule and regret-based rule [2]-[18] as shown inTable 1 .

The first category called network-based rule is defined by the way of using available information in the project, but not information on the resources. It concentrates on shortage processing time rule (SPT); the least activity duration is given the first priority vice versa the most activity duration is given the last priority, as in (1). Further it involves in what is called Most immediate successors (MIS), as in (2), giving the first priority to an activity which is followed by the most immediate successors activities. Another method called Most total successors (MTS), as in (3), is the one which somewhat resembles to the method of MIS. Nevertheless, it involves more in giving the first priority to an activity followed by the most total successors activities. In addition to the methods mentioned above, Greatest Rank Positional Weight (GRPW), as in (4), is also introduced. GRPW concerns the duration of a considerable activity and the duration of its immediate successors activities. The priority of activities is ranked from the most duration of considerable activity and its immediate successor activities to the least duration of considerable activity and its immediate successor activities. The last method of the first category is known as Greatest Rank Positional Weight all (GRPW*), as in (5). It gives the first priority to an activity of which its duration and the duration of its total activities take the longest time.

The second category is critical path-based rule. It relies on information resulted from calculation of critical path method (CPM) based on forward pass and backward pass. The first method in this category namely The Earliest Starting Time (EST), as in (6), is applied for an arrangement of priority order. The first priority of activities is determined on ground of the least earliest starting time. Apart from the earliest time, the earliest finishing time (EFT), as in (7), is also taken into consideration. Not much different from the former, the latter renders the first priority to the least earliest finishing time. The third method is the Earliest Starting Time (Dynamically) (ESTD), as in (8). Similar to the earliest starting time, it gives the first priority to an activity which has the least earliest starting time. However, the difference lies on the fact that it employs the result of the earliest starting time deriving from recalculation of critical path method. Another well-known method is the Earliest Finishing Time (Dynamically) (EFTD), as in (9). In conformity with the Earliest Finishing Time, it gives the first priority to an activity which has the least earliest finishing time, but the difference is that the result of the earliest finishing time comes from recalculation of the same method. The next methods popularly used are the Latest
Starting Time (LST), as in (10), and the latest finishing time (LFT), as in (11). The two give the first priority to an activity which has the least latest starting time and latest finishing time respectively. The last two methods in this second category include the Minimum Slack Time (MSL) and the Minimum Slack Time (Dynamically) (MSLD),as in (12) and (13), respectively. Both methods are similar in giving the first priority to an activity which has the least minimum slack time. However, it is noticeable that while the minimum slack time uses the result of itself based on initial critical path method to arrange priority, the other uses the result of itself based on recalculation of critical path method.

Resource based rule is the third category in this study. This deals with the use of information related to required resources in each activity. The traditional method frequently applied by the researchers is the Greatest Resource Demand (GRD), as in (14). The first priority is determined by an activity demanding the most use of resources. Next, the method called the Weighted Resource Utilization and Precedence (WRUP), as in (15), have been brought into focus. This applies weight multiplier equivalent to 0.7 with the proportion between the required resources and the available resources. In this matter, the first priority is given to an activity gaining the highest result of the above-mentioned calculation.

The fourth category is widely known as the composite rule. The methods of this category are an attempt to overcome the disadvantages of 1-3 categories that only use a set of information. ACROS is considered as the first method of the Composite Rule, as in (16). It employs the total calculation of weight multiplier in daily resource usage of activities within a path connecting the considerable activity to the last activity. The following one is ACTRES, as in (17). It shares certain similarities with ACROS. Anyway, the difference lies on the fact that instead of using daily resource usage in calculation, it replaces with total resource usage. The last two methods consist of TIMROS and TIMRES, as in (18) and (19), respectively. TIMROS, in fact, is developed from ACROS whereas TIMRES is developed from ACTRES. According to the study, the two methods further their calculation on the difference between the latest starting time of the last activity and the considerable activity divided by the difference between the latest starting time of the last activity and the first activity. In addition, the calculation is also based on weight multiplier equivalent to 0.5 . To conclude, the fourth methods as described above gives the first priority to an activity of which the result shows the highest calculation value.

The last category being familiar with researchers in this field is the Regret based rule. This category considers a pair of activities by using dynamic priority rule. It composes of the Improved Resource Scheduling Method (IRSM), the Worst Case Slack Time Rule (WCS), Average Case Slack Time Rule (ACS) and the Worst Case Latest Starting Time (WCLS), as in (20)-(22), (23)-(24), (25) and (26), respectively,. The first three methods are defined as the Partial Schedule Scheme 
(PSS), but the last one is termed as Serial Schedule Scheme (SSS). However, all the mentioned methods assume that an activity proceed temporarily, then the effects of the remaining activities are observed by considering a pair of activities.

The Improved Resource Scheduling Method calculates the effects on the total delay of the earliest starting time from the latest starting time of a pair of activities that results from the considerable activity starting before its pair activity. The Worst Case Slack Time Rule bases its calculation on the minimum value effects of a pair activity starting before the considerable activity on the smallest slack time of the considerable activity. One more recognized method widely used in this category is Average Case Slack Time Rule (ACS). The calculation of this method relies on the average value effects of a pair activity starting before the considerable activity on the smallest slack time of the considerable activity. The last method in the category is Worst Case Latest Starting Time (WCLS) The calculation of the method involves the average value effects of a pair activity starting before the considerable activity on the latest starting time of the considerable activity. In brief, the four methods as described above give the first priority to an activity in which the result shows minimum value.

Although, there are some differences in various methods of heuristic rule, they share the same assumption that each resource has only single skill. However, this assumption is not in conformity with the reality of construction projects containing of some workers who are capable of multi-skills. It is also found that in some occasions when the number of workers is not enough, the workers may be assigned to cope with the unfamiliar activities. Besides, a number of researches in the past demonstrates that the project scheduling assuming that a resource has only single skill causes the inefficient resource utilization. As a result, the project finish time is delayed and the project cost increase unnecessarily.

The approach assuming that a resource has multi-skills has been proposed to bring about efficient resource utilization for project scheduling. Its assumption is based on the fact that a worker is able to work with various skills, so he can adjust himself with anyone of them. The results of several researches concerning this matter maintain that the multi-skilled ability of a worker can contribute to productivity, quality and continuity of work. Also, it leads to the flexibility in work assignment among project managers. Other related researches simultaneously found that the multi-skilled resource approach benefits the workers in term of longer employment duration, better work qualification and increased job satisfaction.

Heuristic method developed by Hegazy et. al. [19] aimed to improve traditional single-skilled resource allocation procedure by allowing insufficient resources to be replaced with other multiskilled resources. For example, two steel workers who have half productivity of formwork can be replaced a carpenter when there is a shortage of carpenters in the construction sites. This method helps in the starting time of a critical activity instead of being delayed which could reduce the project finish time.

AHAMRS proposed by Wongwai et. al. [20] has improved the process of multiskilled resource-constrained scheduling by rearrange the sequence of resource assignment and resource replacement so that the two processes would run more efficiently. This approach also presented the concept of resource-driven task duration where an activity that is not under the team constrained condition can start with partial resource fulfilment in order to increase starting opportunity of activities at earlier time. The results on various case studies demonstrate that it can significantly reduce the project duration.

\section{RESEARCH METHODOLOGY}

A set of instances is necessary in order to compare the performance of the heuristics for multiskilled resource-constrained scheduling. Ten project case studies in these instance sets consist of eight to thirteen activities and four to five renewable multiskilled resources with fixed daily availability are assigned to each activity. Each activity in the projects has known duration and predetermined amount of resources requirement through its duration. The technological relationship between activities has only finish-to-start therefore an activity cannot be started until all its predecessors still not be finished. An activity once started is not be interrupted. To compare the performances of the heuristics, the project case studies were scheduled in limited resource conditions according to twenty-three heuristics shown in Table 1. The simple heuristic algorithm for multiskilled resource scheduling $[19,20]$ where a project is planned under the assumption that each resource can have more than one skill and resource substitution is allowed was applied with project case studies for experimental investigation. For example, assume three units shortage of $\mathrm{R} 1$ occurs and one R1 can be substituted by two R2 or one $R 1$ can be substituted by three R3 ( $2 R 2=1 R 1,3 R 3=1 R 1)$, either six R2 or nine R3 can substitute for the shortage in R1. Furthermore, in case the free amount of either R2 or R3 is not enough to substitute for the whole shortage, a combination of R2 and R3 together, according to their availability, can be used to substitute for the shortage. When a substitution takes place, the resource pool is adjusted accordingly and the resource scheduling procedure is continued without delaying the activity. Nevertheless, if number of qualified resources is not enough to fulfill three units shortage of R1, this activity will be delayed until the earliest day which more resources become available.

\section{RESULTS AND DISCUSSION}

The result of project duration obtained by each heuristic after applying twenty-three heuristics was considered as an indicator to evaluate time performance. Furthermore, the time performance of each heuristic relative to one another was assessed considering the number of times each heuristic gives 
Narongrit Wongwai et al., International Journal of Emerging Trends in Engineering Research, 9(6), June 2021, 633 - 642

Table 1:Heuristics for Resource-Constrained Scheduling

\begin{tabular}{|c|c|}
\hline Heuristics & Priority \\
\hline \multicolumn{2}{|l|}{ Network-Based Rule } \\
\hline Shortage Processing Time (SPT) & $\min d_{j} \quad(1)$ \\
\hline Most Immediate Successors (MIS) & $\max \left|F_{j}\right|(2)$ \\
\hline Most Total Successors (MTS) & $\max \left|F_{j}^{*}\right|(3)$ \\
\hline Greatest Rank Positional Weight (GRPW) & $\max d_{j}+\sum_{i \in F_{j}} d_{i}(4)$ \\
\hline Greatest Rank Positional Weight all (GRPW*) & $\max d_{j}+\sum_{i \in F_{j}^{*}} d_{i}(5)$ \\
\hline \multicolumn{2}{|l|}{ Critical Path-Based Rule } \\
\hline Earliest Starting Time (EST) & $\min E S_{j}(6)$ \\
\hline Earliest Finishing Time (EFT) & $\min E F_{j}(7)$ \\
\hline Earliest Starting Time (Dynamically) (ESTD) & $\min E S_{j}(P S)(8)$ \\
\hline Earliest Finishing Time (Dynamically) (EFTD) & $\min E F_{j}(P S)(9)$ \\
\hline Latest Starting Time (LST) & $\min L S_{j}(10)$ \\
\hline Latest Finishing Time (LFT) & $\min L F_{j}(11)$ \\
\hline Minimum Slack Time (MSL) & $\min T F_{j}(12)$ \\
\hline Minimum Slack Time (Dynamically) (MSLD) & $\min T F_{j}(P S)(13)$ \\
\hline \multicolumn{2}{|l|}{ Resource-Based Rule } \\
\hline Greatest Resource Demand (GRD) & $\max d_{j} \cdot \sum_{r=1}^{m} u_{j r}(14)$ \\
\hline Weighted Resource Utilization and Precedence (WRUP) & $\max \omega \cdot\left|F_{j}\right|+(1-\omega) \cdot \sum_{r=1}^{m} \frac{u_{j r}}{a_{r}} ; \omega=0.7(15)$ \\
\hline \multicolumn{2}{|l|}{ Composite Rule } \\
\hline ACROS & $\max \left\{\sum_{j \in \prod_{j h}} \sum_{r=1}^{m} \frac{u_{j r}}{a_{r}} \mid h=1, \ldots, \pi_{j}\right\}=a_{c r}(16)$ \\
\hline ACTRES & $\max \left\{\sum_{j \in \prod_{j h}} \sum_{r=1}^{m} \frac{u_{j r} \cdot d_{j}}{a_{r}} \mid h=1, \ldots, \pi_{j}\right\}=\operatorname{act}_{j}(17)$ \\
\hline TIMROS & $\max \omega \cdot \frac{\left(L S_{n}-L S_{j}\right)}{\left(L S_{n}-L S_{1}\right)}+(1-\omega) \cdot \frac{a c r_{j}}{a c r_{1}} ; \omega=0.5(18)$ \\
\hline TIMRES & $\max \omega \cdot \frac{\left(L S_{n}-L S_{j}\right)}{\left(L S_{n}-L S_{1}\right)}+(1-\omega) \cdot \frac{a c t_{j}}{a c t_{1}} ; \omega=0.5(19)$ \\
\hline \multicolumn{2}{|l|}{ Regret-Based Rule } \\
\hline Improved Resource Scheduling Method (IRSM) & $\begin{aligned} & \min \max \left\{0,\left[E S_{i}^{r a}(P S, j)-L S_{i}\right] \mid(i, j) \in A P_{n}\right\}(20) \\
& E S_{i}^{r a}(P S, j)=\min \left\{t_{n}+d_{j}, \bar{\Pi}_{(i, j)}\right\}(21) \\
& \bar{\Pi}_{(i, j)}\left\{\begin{array}{c}\infty ;(i, j) \in G F P \\
\prod_{(i, j)} ;(i, j) \in T F P \\
t_{n} ;(i, j) \in C S P\end{array} ;(i, j) \in A P_{n}(22)\right.\end{aligned}$ \\
\hline Worst Case Slack Time Rule (WCS) & $\begin{array}{r}\min L S_{j}-\max \left\{E S_{j}^{r a}(P S, i) \mid(i, j) \in A P_{n}\right\}(23) \\
E S_{j}^{r a}(P S, i)=\min \left\{t_{n}+d_{i}, \bar{\prod}_{(i, j)}\right\}(24)\end{array}$ \\
\hline Average Case Slack Time Rule (ACS) & $\min L S_{j}-\frac{1}{(|E(P S, t)|-1)} \cdot \sum_{i \in(P S, t)-\{j\}} E S_{j}^{r a}(P S, i)(25)$ \\
\hline Worst Case Latest Starting Time (WCLS) & $\min \min \left\{L S_{j}^{r a}(P S, i)\right\}(26)$ \\
\hline
\end{tabular}


Narongrit Wongwai et al., International Journal of Emerging Trends in Engineering Research, 9(6), June 2021, 633 - 642

the shortest project duration. Basically, the heuristics with better time performance have (1) a lower project duration, (2) a higher number of times producing the shortest project duration and (3) the worst duration (longest duration) less frequently.

Table 2presents that TIMROS and TIMRES produce the shortest total project duration (406 days) followed by ACROS, WCS, ACS (409 days) and ACTRES (411 days), respectively. As can be noticed that, TIMROS, TIMRES, ACROS and ACTRESS are composite rule category while WCS and ACS are regret based rule. The heuristics that performed the worst were GRD, MIS, WRUP and GRPW, respectively. GRD and WRUP are resource-based rule category while MIS and GRPW are network-based rule category. It should be pointed out that the heuristics dealing with the use of several information outperformed the others e.g. TIMROS and GRD where both of daily resource usage and time information of each activity are together taken into consideration, as in(27)-(29). Furthermore, it is noticeable that the heuristics considering information throughout the path connecting from the considerable activity to the late activity can produce the lower project duration. In equation 1 and 2, TIMROS employs the total calculation of weight multiplier equivalent to 0.5 on the difference between the latest starting time of the last activity and the considerable activity divided by the difference between the latest starting time of the last activity and the first activity i.e. the higher proportional, the longer following path. In addition, it determines the daily resource usage of activities within a path connecting the considerable activity to the last activity divided by the first activity to the last activity i.e. the higher proportional, the higher remaining resource utilization. Whereas, in equation 3, GRD deals with the use of information related to required resources only in considerable activity during its duration regardless of the resource requirement of its following activities.

$$
\begin{aligned}
& \mathrm{P}_{\mathrm{j}}(\text { TIMROS })=\omega \cdot \frac{\left(L S_{n}-L S_{j}\right)}{\left(L S_{n}-L S_{1}\right)}+(1-\omega) \cdot \frac{a c r_{j}}{a c r_{1}} ; \omega=0.5 \\
& \operatorname{acr}_{j}=\max \left\{\sum_{j \in \prod_{j h}} \sum_{r=1}^{m} \frac{u_{j r}}{a_{r}} \mid h=1, \ldots, \pi_{j}\right\} \\
& \mathrm{P}_{\mathrm{j}}(\mathrm{GRD})=d_{j} \cdot \sum_{r=1}^{m} u_{j r} \\
& \text { Where } \\
& \mathrm{P}_{\mathrm{j}}(\text { TIMROS })=\text { priority value calculated by TIMROS; } \\
& \mathrm{P}_{\mathrm{j}}(\mathrm{GRD}) \quad=\text { priority value calculated by GRD; } \\
& \omega \quad=\text { weight multiplier equivalent to } 0.5 \text {; } \\
& L S_{n} \quad=\text { latest starting time of the last activity n; } \\
& L S_{j} \quad=\text { latest starting time of considerable activity } \mathrm{j} \text {; } \\
& L S_{1} \quad \text { =latest starting time of the first activity; } \\
& \prod_{j h} \quad=\text { set of the path connecting activity } \mathrm{j} \text { to the last } \\
& \text { activity } \mathrm{n} \text {; } \\
& \pi_{j} \quad=\text { total number of the path connecting activity } \mathrm{j} \\
& \text { to the last activity n; } \\
& u_{j r} \quad=\text { daily resource usage of resource type } \mathrm{r} \text { for } \\
& \text { activity j; } \\
& a_{r} \quad=\text { daily available resource type } \mathrm{r} \text {; } \\
& d_{j} \quad=\text { duration of activity } \mathrm{j} \text {. }
\end{aligned}
$$

The performance of the heuristics was also evaluated in term of the percentage increase of project duration $(\mathrm{X})$ above the minimum project duration $\left(\mathrm{X}_{\min }\right)$ obtained by each heuristic given in Table 3. Due to the high consistency of the shortest schedule among the top heuristics, the test found evidence that either TIMROS or TIMRES obtained the best results. Both of them produces the zero-percentage increase for every project case studies. Furthermore, the slight difference in the percentage increase $(8.8 \%)$ among the top four heuristics are WCS and ACS. As can be noticed that, TIMROS, TIMRES, WCS and TIMRES not only outperformed in the shortest schedule but also in the consistency rate. In order to test a higher number of times producing the shortest project duration (the frequency rate) the results of total project duration in Table 2obtained by difference heuristics were arranged in ascending order.

Table 4 clearly presents that TIMROS and TIMRES produced the shortage schedule more frequently than any other heuristic (100\%). Furthermore, they were found that to find the worst duration (longest duration) less frequently than other heuristics. Although WCS, ACS, MIS and GRPW found the shortage duration the same number of time (80\%), WCS and ACS produced the lower percentage increase $(8.8 \%)$ above the minimum project duration in Table 3.Furthermore, Table 2 presents that MIS was the twelfth total project duration among the other heuristics for the reason that MIS underperformed on the seventh project in which the network complexity (the average number of successor relationships per activity) and the standard deviation were very high.

Table 5 presents the average of project duration classified into five categories: network-based rule, critical path-based rule, composite rule and regret-based rule. Since overall, the top four heuristics: TIMROS, TIMRES, ACROS and ACTRESS were found to (1) have the lower project duration, (2) find the shortest duration more frequently and (3) find the worst duration (longest duration) less frequently were classified into the composite rule, it accordingly produced the lowest average of project duration. Furthermore, regret-based rule was the second category with the lower average of project duration. As can be noticed that, both of above mentioned categories relied on several information outperformed other categories based on single information. As can be observed in Table 2, WCLS obstructed the overall performance of regret-based rule. It is also found that WCLS termed as Serial Schedule Scheme (SSS) underperform the remaining heuristics: IRSM, WCS and ACS defined as the Partial Schedule Scheme (PSS).

As in Figure 1, the statistical analysis applied with the normal distribution assumption found that the results of project duration obtained by the top two heuristics: TIMROS and TIMRES lie within minus two standard deviation $(-2 \sigma)$ of the mean $(\overline{\mathrm{X}})$. Furthermore, the following heuristics: SPT, EST, EFT, ESTD, EFTD, LFT, ACROS, ACTRES, IRSM and ACS produced slight difference results within minus one standard deviation $(-1 \sigma)$ of the mean $(\overline{\mathrm{X}})$. Since most of aforementioned heuristics were relied on several information, therefore this criterion has a significant effect on reducing project duration by approximately 1-2 times of the standard deviation. 
Narongrit Wongwai et al., International Journal of Emerging Trends in Engineering Research, 9(6), June 2021, $633-642$

Table 2:Project Duration

\begin{tabular}{|c|c|c|c|c|c|c|c|c|c|c|c|}
\hline \multirow{2}{*}{ Heuristics } & \multicolumn{10}{|c|}{ Project Duration (day) } & \multirow{2}{*}{ Total } \\
\hline & 1 & 2 & 3 & 4 & 5 & 6 & 7 & 8 & 9 & 10 & \\
\hline \multicolumn{12}{|l|}{ Network-Based Rule } \\
\hline SPT & 17 & 25 & 17 & 19 & 37 & 19 & 59 & 73 & 73 & 73 & 412 \\
\hline MIS & 16 & 25 & 18 & 18 & 37 & 19 & 73 & 73 & 72 & 73 & 424 \\
\hline MTS & 17 & 25 & 18 & 18 & 37 & 19 & 61 & 73 & 81 & 73 & 422 \\
\hline GRPW & 17 & 25 & 17 & 18 & 37 & 19 & 72 & 73 & 72 & 73 & 423 \\
\hline GRPW* & 17 & 25 & 17 & 18 & 37 & 20 & 61 & 73 & 81 & 73 & 422 \\
\hline \multicolumn{12}{|c|}{ Critical Path-Based Rule } \\
\hline EST & 16 & 25 & 18 & 18 & 37 & 19 & 59 & 75 & 72 & 73 & 412 \\
\hline EFT & 17 & 25 & 17 & 19 & 37 & 19 & 59 & 73 & 73 & 73 & 412 \\
\hline ESTD & 16 & 25 & 18 & 19 & 37 & 19 & 60 & 73 & 72 & 73 & 412 \\
\hline EFTD & 17 & 25 & 17 & 19 & 37 & 19 & 59 & 73 & 73 & 73 & 412 \\
\hline LST & 16 & 25 & 17 & 18 & 37 & 20 & 58 & 73 & 81 & 73 & 418 \\
\hline LFT & 16 & 25 & 17 & 19 & 37 & 19 & 62 & 75 & 72 & 73 & 415 \\
\hline MSL & 16 & 25 & 17 & 18 & 37 & 21 & 58 & 73 & 81 & 73 & 419 \\
\hline MSLD & 16 & 25 & 17 & 18 & 37 & 20 & 58 & 73 & 81 & 73 & 418 \\
\hline \multicolumn{12}{|l|}{ Resource-Based Rule } \\
\hline GRD & 17 & 25 & 17 & 19 & 37 & 19 & 72 & 75 & 73 & 73 & 427 \\
\hline WRUP & 17 & 25 & 17 & 18 & 37 & 20 & 72 & 73 & 72 & 73 & 424 \\
\hline \multicolumn{12}{|l|}{ Composite Rule } \\
\hline ACROS & 17 & 25 & 17 & 19 & 37 & 20 & 56 & 73 & 72 & 73 & 409 \\
\hline ACTRES & 17 & 25 & 17 & 19 & 37 & 20 & 56 & 75 & 72 & 73 & 411 \\
\hline TIMROS & 16 & 25 & 17 & 18 & 37 & 19 & 56 & 73 & 72 & 73 & 406 \\
\hline TIMRES & 16 & 25 & 17 & 18 & 37 & 19 & 56 & 73 & 72 & 73 & 406 \\
\hline \multicolumn{12}{|l|}{ Regret-Based Rule } \\
\hline IRSM & 16 & 25 & 17 & 19 & 37 & 19 & 61 & 75 & 72 & 73 & 414 \\
\hline WCS & 16 & 25 & 17 & 18 & 37 & 20 & 58 & 73 & 72 & 73 & 409 \\
\hline ACS & 16 & 25 & 17 & 18 & 37 & 20 & 58 & 73 & 72 & 73 & 409 \\
\hline WCLS & 16 & 25 & 17 & 18 & 37 & 20 & 60 & 73 & 81 & 73 & 420 \\
\hline Minimum & 16 & 25 & 17 & 18 & 37 & 19 & 56 & 73 & 72 & 73 & 406 \\
\hline Maximum & 17 & 25 & 18 & 19 & 37 & 21 & 73 & 75 & 81 & 73 & 427 \\
\hline Standard Deviation & 0.5 & - & 0.4 & 0.5 & - & 0.6 & 5.5 & 0.8 & 4.0 & - & 6.2 \\
\hline
\end{tabular}


Narongrit Wongwai et al., International Journal of Emerging Trends in Engineering Research, 9(6), June 2021, $633-642$

Table 3:The difference percentage $(\%)$ between $X$ and Xmin of multiskilled resource scheduling

\begin{tabular}{|c|c|c|c|c|c|c|c|c|c|c|c|}
\hline \multirow{2}{*}{ Heuristic Rule } & \multicolumn{10}{|c|}{ The difference percentage $(\%)$ between $X$ and $X_{\min }$} & \multirow{2}{*}{ Total } \\
\hline & 1 & 2 & 3 & 4 & 5 & 6 & 7 & 8 & 9 & 10 & \\
\hline \multicolumn{12}{|l|}{ Network-Based Rule } \\
\hline SPT & 6.3 & 0.0 & 0.0 & 5.6 & 0.0 & 0.0 & 5.4 & 0.0 & 1.4 & 0.0 & 18.6 \\
\hline MIS & 0.0 & 0.0 & 5.9 & 0.0 & 0.0 & 0.0 & 30.4 & 0.0 & 0.0 & 0.0 & 36.2 \\
\hline MTS & 6.3 & 0.0 & 5.9 & 0.0 & 0.0 & 0.0 & 8.9 & 0.0 & 12.5 & 0.0 & 33.6 \\
\hline GRPW & 6.3 & 0.0 & 0.0 & 0.0 & 0.0 & 0.0 & 28.6 & 0.0 & 0.0 & 0.0 & 34.8 \\
\hline GRPW* & 6.3 & 0.0 & 0.0 & 0.0 & 0.0 & 5.3 & 8.9 & 0.0 & 12.5 & 0.0 & 32.9 \\
\hline \multicolumn{12}{|c|}{ Critical Path-Based Rule } \\
\hline EST & 0.0 & 0.0 & 5.9 & 0.0 & 0.0 & 0.0 & 5.4 & 2.7 & 0.0 & 0.0 & 14.0 \\
\hline EFT & 6.3 & 0.0 & 0.0 & 5.6 & 0.0 & 0.0 & 5.4 & 0.0 & 1.4 & 0.0 & 18.6 \\
\hline ESTD & 0.0 & 0.0 & 5.9 & 5.6 & 0.0 & 0.0 & 7.1 & 0.0 & 0.0 & 0.0 & 18.6 \\
\hline EFTD & 6.3 & 0.0 & 0.0 & 5.6 & 0.0 & 0.0 & 7.1 & 0.0 & 0.0 & 0.0 & 18.6 \\
\hline LST & 0.0 & 0.0 & 0.0 & 0.0 & 0.0 & 5.3 & 3.6 & 0.0 & 12.5 & 0.0 & 21.3 \\
\hline LFT & 0.0 & 0.0 & 0.0 & 5.6 & 0.0 & 0.0 & 10.7 & 2.7 & 0.0 & 0.0 & 19.0 \\
\hline MSL & 0.0 & 0.0 & 0.0 & 0.0 & 0.0 & 10.5 & 3.6 & 0.0 & 12.5 & 0.0 & 26.6 \\
\hline MSLD & 0.0 & 0.0 & 0.0 & 0.0 & 0.0 & 5.3 & 3.6 & 0.0 & 12.5 & 0.0 & 21.3 \\
\hline \multicolumn{12}{|l|}{ Resource-Based Rule } \\
\hline GRD & 6.3 & 0.0 & 0.0 & 5.6 & 0.0 & 0.0 & 28.6 & 2.7 & 1.4 & 0.0 & 44.5 \\
\hline WRUP & 6.3 & 0.0 & 0.0 & 0.0 & 0.0 & 5.3 & 28.6 & 0.0 & 0.0 & 0.0 & 40.1 \\
\hline \multicolumn{12}{|l|}{ Composite Rule } \\
\hline ACROS & 6.3 & 0.0 & 0.0 & 5.6 & 0.0 & 5.3 & 0.0 & 0.0 & 0.0 & 0.0 & 17.1 \\
\hline ACTRES & 6.3 & 0.0 & 0.0 & 5.6 & 0.0 & 5.3 & 0.0 & 2.7 & 0.0 & 0.0 & 19.8 \\
\hline TIMROS & 0.0 & 0.0 & 0.0 & 0.0 & 0.0 & 0.0 & 0.0 & 0.0 & 0.0 & 0.0 & 0.0 \\
\hline TIMRES & 0.0 & 0.0 & 0.0 & 0.0 & 0.0 & 0.0 & 0.0 & 0.0 & 0.0 & 0.0 & 0.0 \\
\hline \multicolumn{12}{|l|}{ Regret-Based Rule } \\
\hline IRSM & 0.0 & 0.0 & 0.0 & 5.6 & 0.0 & 0.0 & 8.9 & 2.7 & 0.0 & 0.0 & 17.2 \\
\hline WCS & 0.0 & 0.0 & 0.0 & 0.0 & 0.0 & 5.3 & 3.6 & 0.0 & 0.0 & 0.0 & 8.8 \\
\hline ACS & 0.0 & 0.0 & 0.0 & 0.0 & 0.0 & 5.3 & 3.6 & 0.0 & 0.0 & 0.0 & 8.8 \\
\hline WCLS & 0.0 & 0.0 & 0.0 & 0.0 & 0.0 & 5.3 & 7.1 & 0.0 & 12.5 & 0.0 & 24.9 \\
\hline
\end{tabular}


Narongrit Wongwai et al., International Journal of Emerging Trends in Engineering Research, 9(6), June 2021, 633 - 642

Table 4: The frequency rate of producing the shortest project duration

\begin{tabular}{|c|c|c|c|c|}
\hline \multirow{2}{*}{ Heuristic Rule } & \multicolumn{4}{|c|}{ Frequency } \\
\hline & $1^{\text {st }}$ rank & $2^{\text {nd }}$ rank & $3^{\text {rd }}$ rank & $>3^{\text {rd }}$ rank \\
\hline SPT & $60 \%$ & $30 \%$ & $10 \%$ & - \\
\hline MIS & $80 \%$ & $10 \%$ & - & $10 \%$ \\
\hline MTS & $60 \%$ & $20 \%$ & $10 \%$ & $10 \%$ \\
\hline GRPW & $80 \%$ & $10 \%$ & - & $10 \%$ \\
\hline GRPW* & $60 \%$ & $20 \%$ & $10 \%$ & $10 \%$ \\
\hline EST & $70 \%$ & $20 \%$ & $10 \%$ & - \\
\hline EFT & $60 \%$ & $30 \%$ & $10 \%$ & - \\
\hline ESTD & $70 \%$ & $20 \%$ & - & $10 \%$ \\
\hline EFTD & $60 \%$ & $30 \%$ & $10 \%$ & - \\
\hline LST & $70 \%$ & $20 \%$ & $10 \%$ & - \\
\hline LFT & $70 \%$ & $20 \%$ & - & $10 \%$ \\
\hline MSL & $70 \%$ & $10 \%$ & $20 \%$ & - \\
\hline MSLD & $70 \%$ & $20 \%$ & $10 \%$ & - \\
\hline GRD & $50 \%$ & $40 \%$ & - & $10 \%$ \\
\hline WRUP & $70 \%$ & $20 \%$ & - & $10 \%$ \\
\hline ACROS & $70 \%$ & $30 \%$ & - & - \\
\hline ACTRES & $60 \%$ & $40 \%$ & - & - \\
\hline TIMROS & $100 \%$ & - & - & - \\
\hline TIMRES & $100 \%$ & - & - & - \\
\hline IRSM & $70 \%$ & $20 \%$ & - & $10 \%$ \\
\hline WCS & $80 \%$ & $20 \%$ & - & - \\
\hline ACS & $80 \%$ & $20 \%$ & - & - \\
\hline WCLS & $70 \%$ & $10 \%$ & $10 \%$ & $10 \%$ \\
\hline
\end{tabular}

Table 5: Average of project duration

\begin{tabular}{|c|c|}
\hline Categories of Heuristic Rule & Average (day) \\
\hline Network-Based Rule & 420.6 \\
\hline Critical Path-Based Rule & 414.8 \\
\hline Resource-Based Rule & 425.5 \\
\hline Composite Rule & 408.0 \\
\hline Regret-Based Rule & 413.0 \\
\hline
\end{tabular}


Conversely, MIS, MTS, GRPW, GRPW*, GRD and WRUP relied on the only one information produced the worst results, i.e., their results lied within plus two standard deviation $(+2 \sigma)$ of the mean $(\overline{\mathrm{X}})$.

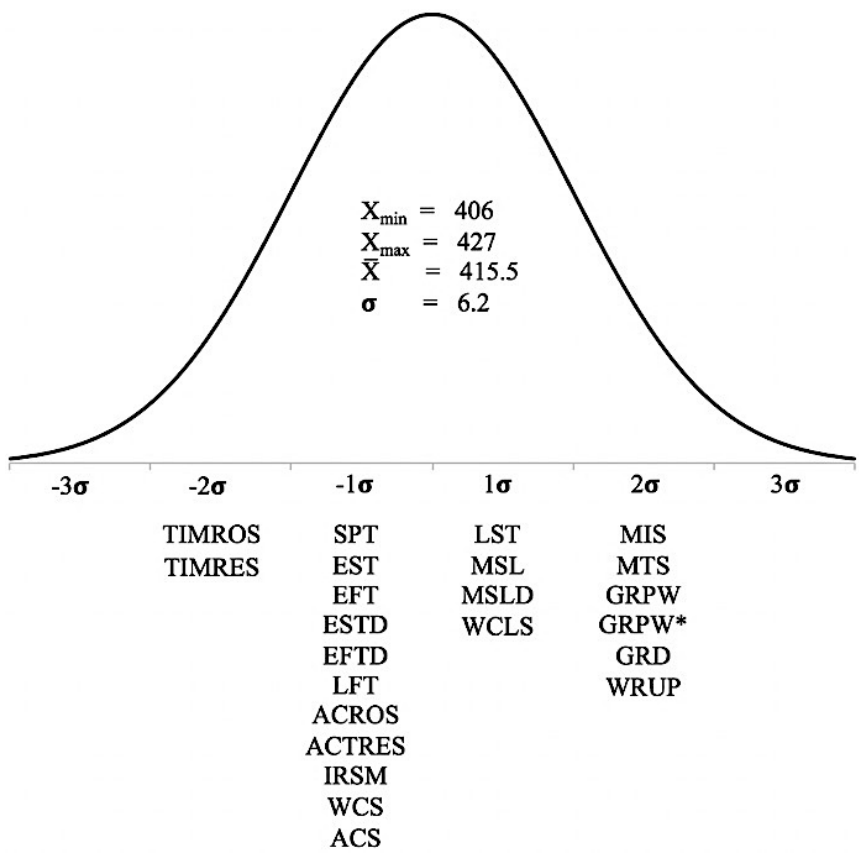

Figure 1: The Statistical Analysis of Project Duration

\section{CONCLUSIONS}

This study explored the performance of the existing heuristics for multiskilled resource-constrained scheduling. The results found that the heuristics with good performance for multiskilled resource scheduling are TIMROS, TIMRES, ACROS, WCS ACS and ACTRES. Overall, they outperform the others in shortening the project duration. It should be pointed out that the heuristics dealing with the use of several information are likely better to get shorter project duration. There are enough statistical evidences to conclude that their criterions have a significant effect on reducing project duration by approximately 1-2 times of the standard deviation. The top four heuristics: TIMROS, TIMRES, ACROS and ACTRESS were classified into the composite rule, it accordingly produced the lowest average of project duration.

This study recommends using either TIMROS or TIMRES as the rule for prioritizing the eligible activities which demand the same multiskilled resources at the same time since they are not only to produce a higher number of times producing the shortest project duration but also the worst duration (longest duration) less frequently. It is also found that Serial Schedule Scheme (SSS) underperform the Partial Schedule Scheme (PSS) e.g. WCLS shows the worst performance among the heuristics within the regret-based rule.

\section{REFERENCES}

1. E. Demeulemeester, and W. Herroelen. A Branch-and-Bound Procedure for the Multiple Resource-Constrained Project Scheduling Problem, Management Science, vol. 38, no. 12, pp. 1803-1818, 1992.

2. Alvarez-Valdes, R. and J.M. Tamarit. Heuristic Algorithms for Resource-Constrained Project Scheduling: A Review and an Empirical Analysis, in Slowinski, R. and J. Weglarz (Eds.): Advances in Project Scheduling, Elsevier, Amsterdam, 1989, pp. 113-134.

3. D.O. Bedworth. Industrial Systems: Planning, Analysis, Control; Ronald Press: New York, 1973.

4. J.D. Brand, W.L. Meyer, and L.R. Shaffer. The Resource Scheduling Method for Construction. Civil Engineering Studies Report No.5; University of Illinois, 1964.

5. P. Brucker, A. Drexl, R. Mohring, K. Neumann, and E. Pesch. Resource-constrained project scheduling: Notation, classification, models, and methods, European Journal of Operational Research, vol. 112, no. 1, pp. 3-41, 1999.

6. E.W. Davis, and J.H. Patterson. A Comparison of Heuristic and Optimum Solutions in Resource-Constrained Project Scheduling,Management Science, vol. 21, pp. 944-955, 1975.

7. E.A. Elsayed, and N.Z. Nasr. Heuristics for Resource-Constrained Project Scheduling, International Journal of Production Research, vol. 24, pp. 299-310, 1986.

8. S. Hartmann, and R. Kolisch. Experimental evaluation of state-of-the-art heuristics for the resource-constrained project scheduling problem, European Journal of Operational Research, vol. 127, pp. 394-407, 2000.

9. W. Herroelen, E. Demeulemeester, and B. De Reyck.Resource- constrained project scheduling: A survey of recent developments,Computers \&Operations Research, vol. 25, no. 4, pp. 279-302, 1998.

10. O. Icmeli, S. SelcukErenguc, and Ch.1. Zappe.Project Scheduling Problems: A Survey,International Journal of Operations \& Production Management, vol.13, pp. 80-91, 1993.

11. R. Klein. Bidirectional Planning: Improving Priority Rule-Based Heuristics for Scheduling Resource-Constrained Projects, European Journal of Operational Research, vol. 127, pp. 619-638, 2000.

12. R. Klein. Scheduling of Resource-Constrained Projects, Operations Research/Computer Science, Interfaces Series 10, 2000, pp. 181-187.

13. R. Kolisch, and S. Hartmann. Experimental investigation of heuristics for resource-constrained project scheduling: An update, European Journal of Operational Research, 2005, forthcoming.

14. R. Kolisch, and R. Padman. An integrated survey of deterministic project scheduling, OMEGA 29, 2001, pp. 249-272. 
15. R. Kolisch. Efficient Priority Rule for the Resource-Constrained Project Scheduling Problem, Journal of Operations Management, vol. 14, pp. 179-192, 1996.

16. R. Kolisch. Project Scheduling under Resource Constraints - Efficient Heuristics for Several Problem Classes, Physica: Heidelberg, 1995.

17. P.R. Thomas, and S. Salhi. An Investigation into the Relationship of Heuristic Performance with Network-Resource Characteristics, Journal of the Operational Research Society, vol. 48, pp. 34-43, 1997.

18. G. Ulusoy, and L.Ozdamar. Heuristic Performance and Network/Resource Characteristics in Resource-Constrained Project Scheduling, Journal of the Operational Research Society, vol. 40, pp. 1145-1152, 1989.

19. T. Hegazy, A. Shabeeb, R. El-beltagi, and T. Cheema. Algorithm for scheduling with multi-skilled construction resources, Journal of Construction Engineering and Management, vol. 126, no. 6, pp. 125-133, 2000.

20. N. Wongwai, and S. Malaikrisanachalee, Augmented Heuristic Algorithm for Multi-skilled Resource Scheduling, Automation in Construction, vol. 20, no. 4, pp. 429-445, 2011. 